\title{
Article
}

\section{How Active Inference Could Help Revolutionise Robotics}

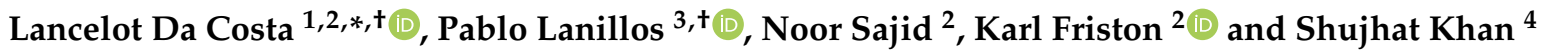 \\ 1 Department of Mathematics, Imperial College London, London SW7 2AZ, UK \\ 2 Wellcome Centre for Human Neuroimaging, University College London, London WC1N 3AR, UK \\ noor.sajid.18@ucl.ac.uk (N.S.); k.friston@ucl.ac.uk (K.F.) \\ 3 Department of Artificial Intelligence, Donders Institute for Brain, Cognition and Behavior, Radboud University, \\ 6525 XZ Nijmegen, The Netherlands; p.lanillos@donders.ru.nl \\ 4 Milton Keynes Hospital, Oxford Deanery, Milton Keynes MK6 5LD, UK; shujhat.khan15@imperial.ac.uk \\ * Correspondence: 1.da-costa@imperial.ac.uk \\ + These authors contributed equally to this work.
}

Citation: Da Costa, L.; Lanillos, P.; Sajid, N.; Friston, K.; Khan, S. How

Active Inference Could Help

Revolutionise Robotics. Entropy 2022,

24, 361. https://doi.org/10.3390/

e24030361

Academic Editor: Udo Von Toussaint

Received: 21 January 2022

Accepted: 28 February 2022

Published: 2 March 2022

Publisher's Note: MDPI stays neutral with regard to jurisdictional claims in published maps and institutional affiliations.

Copyright: (C) 2022 by the authors. Licensee MDPI, Basel, Switzerland. This article is an open access article distributed under the terms and conditions of the Creative Commons Attribution (CC BY) license (https:// creativecommons.org/licenses/by/ $4.0 /)$.

\begin{abstract}
Recent advances in neuroscience have characterised brain function using mathematical formalisms and first principles that may be usefully applied elsewhere. In this paper, we explain how active inference-a well-known description of sentient behaviour from neuroscience-can be exploited in robotics. In short, active inference leverages the processes thought to underwrite human behaviour to build effective autonomous systems. These systems show state-of-the-art performance in several robotics settings; we highlight these and explain how this framework may be used to advance robotics.
\end{abstract}

Keywords: free energy; model-based control; adaptive robots; generative model; Bayesian inference; filtering; neurotechnology

\section{Active Inference}

Active inference (AIF) is a unifying framework for describing and designing adaptive systems [1-4]. AIF emerged in the late 2000s as a unified theory of brain function [5,6] derived from statistical physics $[2,7]$ and has since been used to simulate a wide range of behaviours in neuroscience [1,8], machine learning [9-13] and robotics [14]. AIF is an interesting framework for robotics because it unifies state-estimation, control and world model learning as inference processes that are solved by optimising a single objective functional: a free energy (also known as negative evidence lower bound), as used in variational Bayesian inference [15]. Furthermore, it endows robots with adaptive capabilities central to real world applications [14] (e.g., adaptation to internal and external parameter changes [16]). Additionally, its strong neuroscience foundation reduces the gap between engineering and the life sciences, thereby finessing human-centred robotic applications.

Although AIF has yet to be scaled-to tackle high dimensional problems-to the same extent as established approaches, such as deep reinforcement learning [17,18], numerical analyses generally show that active inference performs at least as well in simple environments [9,19-23], and better in environments featuring volatility, ambiguity and context sensitivity [21,22]. In this paper, we consider how AIF's features could help address key technical challenges in robotics and discuss practical robotic applications. Our exposition provides a broad perspective that suppresses mathematical details, which can be found in the references herein $[1-4,14,24,25]$.

In AIF, a generative model encodes an agent's predictions (i.e., posterior beliefs), and preferred state and observation trajectories (i.e., prior beliefs) [2]. Behaviour realises the agent's preferences by matching posterior with prior beliefs. Specifically, state-estimation, control and learning are unified by minimising a free energy functional scoring the discrepancy between current beliefs and prior preferences under the state-space model. For 
continuous states, AIF filters incoming observations through variational inference in generalised coordinates of motion [26]. This enables flexible and scalable inference algorithms and extends Kalman filters by accommodating non-linear, non-Markovian time-series [26-28]. AIF generalises discrete and continuous optimal control [29], and planning to partially observed environments, similarly to model predictive control or control as inference [30,31]. However, a crucial difference is that the (expected) free energy optimised during planning combines exploitative and explorative behaviour [32] in a Bayes optimal fashion [2,7]. The agent's model-i.e., representations and goals-can then be learnt through few-shot learning [21], structure learning, imitation learning, and evolutionary approaches [1,33-35].

\section{Solutions to Technical Challenges in Robotics}

Current AIF models can help address challenges that require online adaptation, robustness and explainability, and may bring new perspectives to the state-of-the-art in estimation, control and planning-see Figure 1.

- Accurate and robust state tracking. Filtering schemes developed for neuroimaging time-series [26] enable accurate state-tracking in highly complex and volatile environments [27,36]. This allows for continuous refinement of past, present, future state-estimation and the estimated precision of sensors as new information arrives [37] (c.f., Bayes optimal estimators of Kalman gain [38]). Moreover, AIF fuses multiple sensory streams, by weighing incoming sensory information by their estimated precision $[36,39]$. This enables accurate and robust inferences.

- Adaptive model-based and shared control. Describing the agent's behaviour with a generative model-prescribing attracting states and trajectories-ensures robustness and adaptivity in the presence of noise, external fluctuations, and parameter changes. AIF humanoid robots [36] and industrial manipulators [40] show improved behaviour in the presence of internal and external parameter changes [16] and shared compliance control [41]. The robot's autonomy-in shared control-can also be dynamically tuned. In particular, the operator may be given high-level control and the robot low-level control.

- Learning and grounding. AIF agents learn from sparse and noisy observations by actively sampling informative data points, enabling few-shot learning. Learning latent structure by optimising model evidence, subject to prior preferences in the generative model, leads to organising knowledge in hierarchical, sparsely interconnected modular (i.e., factorised) representations with temporal depth, usually represented with a graphical model [2]. This offers a promising pathway for biologically plausible neurosymbolic technologies [42,43].

- Operational specification, safety and explainability. AIF behaviour is explainable as a mixture of information and goal-seeking policies that are explicitly encoded (and evaluated in terms of expected free energy) in the generative model as priors-which can be specified by the user. Planning, which proceeds by generating counterfactual actions and assessing their consequences [1], can be monitored online and control can be returned to the user if necessary (i.e., policy switching). Moreover, the generative model can be specified as a directed graph (i.e., a Bayesian network), which entails the causal relationships between agent's representations $[44,45]$. This affords an explicit and transparent explanation of sentient behaviour. 


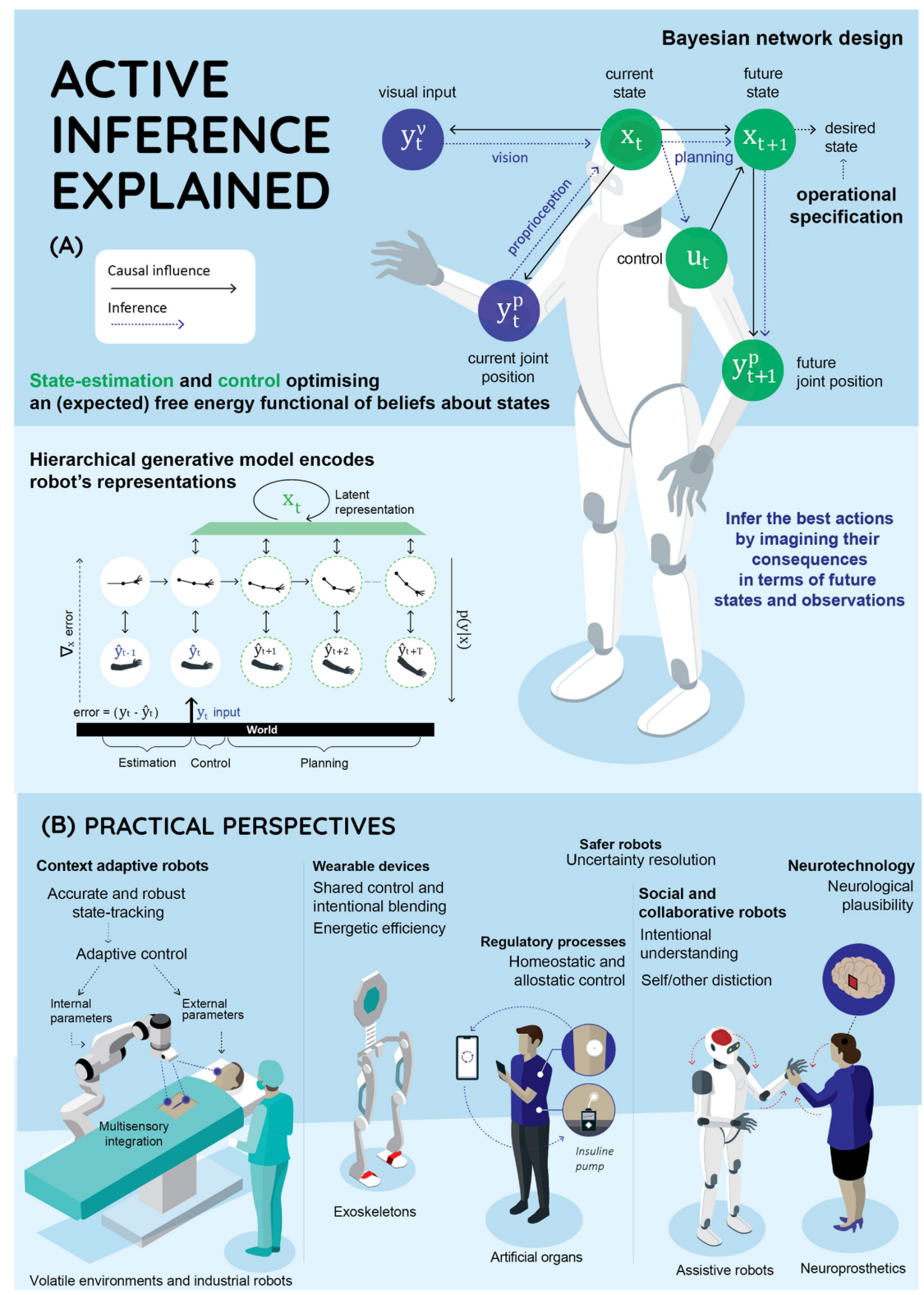

Figure 1. Active inference framework (AIF). AIF could engender important advances in estimation, control, planning and learning in robotics with applications including social, industrial and collaborative robotics, body prosthetics and neurotechnology. (A) AIF explained: Blue circles indicate observations while grey circles indicate random variables that need to be inferred. The black arrows indicate causal relationships implicit in a graphical model (e.g., a Bayesian network). The blue arrows indicate the process by which the agent infers future actions and observations. First, the agent infers the current states from available observation modalities (Bayesian fusion). Then, the agent infers the best available course of action by imagining the counterfactual consequences, in terms of future states and observations. These inferential processes are solved by optimising an (expected) free energy functional of beliefs about states and plausible action sequences. AIF generative models may be hierarchical and encode agent's representations at increasing levels of abstraction and temporal scales. Perception minimises the discrepancy between predictions and input at all levels. The top layer encodes the estimated (and preferred) states of the world-and the bottom layer encodes sensory input. (B) Practical perspectives: AIF can provide context sensitivity, online adaptivity, accurate state tracking, uncertainty resolution and shared control in a neurologically plausible fashion throughout a wide range of applications. 


\section{Practical Perspectives}

Based on these properties, we envisage important applications of AIF in robotics.

- Context adaptive robots. AIF agents build generative (world) models by continuously optimising free energy with regard to incoming data. This optimisation process maximises model accuracy while minimising complexity, which enables generalisation and context-adaptivity [36]. Contrariwise, robots that solely optimise accuracy risk overfitting, which could lead to catastrophic outcomes when the context changes, such as when performing assistive surgery on a new patient. The ability to generalise and adapt is necessary for robotic skills such as scene understanding and adaptive control and should facilitate robots to operate in volatile (e.g., social) environments (e.g., hospitals) [36,46]. In industrial applications, this allows robots to operate freely while adapting to real world conditions-once the designer has specified preferences over the final outcome.

- Safer robots. AIF agents continuously resolve uncertainty by selecting informative actions that minimise risk [1], which is important for high-stakes, high-uncertainty tasks, such as human-robot interaction [41]. Actions are selected to minimise expected free energy, which minimises risk (expected cost) and ambiguity (expected inaccuracy) [1]. This allows for information seeking behaviour that is accompanied with an explicit and quantifiable measure of risk. Additionally, when uncertain about current states of affair, robots should automatically seek advice and guidance from the user, e.g., via shared control.

- Social and collaborative robots. AIF robots model others' intentions to predict others' actions, such as movements [47], enabling intentional understanding [48]. This allows robots to operate safely in social environments by constantly resolving uncertainty about others' intentions and implicit goals [42]. This embodiment [49] is crucial for social robots, such as personal aides, auxiliary robot nurses and companions, e.g., assisting the disabled and elderly. In collaborative robotics, AIF allows for imitation learning and intentional blending, whence robot goals and intentions can be guided by the user before and during the task $[41,50]$.

- Wearable devices. The belief updating process that underwrites AIF is energetically efficient [51], which should aid the development of wearable devices with a degree of autonomy, such as exoskeletons [52]. This follows as optimising model free energy decreases the movement from prior to posterior, which corresponds to the computational (and hence energetic) cost of inference [1,2]. In addition, wearables directed by human intention [53] should benefit from AIF's intentional understanding [48], and adaptive and shared control capabilities [41].

- Regulatory processes. Generative models with temporal depth induce allostatic control, whence the robot acts on its environment to pre-empt homeostatic control $[54,55]$. This should benefit regulatory processes subject to strong external perturbations $[16,36]$, such as closed-loop medical applications such as artificial organs (e.g., the artificial pancreas).

- Neurotechnology. The neurological functional plausibility of specific AIF algorithms $[1,46,56]$ should facilitate integration with the nervous system. This opens new opportunities for neurotechnology, BCI-enabled sensorimotor restoration, perceptual body extension and brain or body enhancement using prosthetics and implants [57]. Currently, AIF provides testable hypotheses for optimising neural excitatory-inhibitory balance using deep brain stimulation to alleviate functional deficits induced by brain lesions [58]. Soon, monitoring of brain activity may predict aberrant neural responses, such as seizures, and anticipate the required intervention.

\section{Discussion}

In this perspective, we explained how active inference-a framework for describing and designing adaptive systems originating in computational neuroscience-can be exploited in robotics. In particular, we surveyed some key features of AIF that could 
provide solutions to current technical challenges in robotics, and how these could benefit human-centred robotic applications in the short-term.

In brief, the theoretical foundations of AIF suggest the potential for important advances in state-estimation, control, planning and learning that undergirds autonomous robots. This suggests a promising avenue for endowing robots with online adaptive strategies and context-sensitive and explainable decision-making. In turn, these advances could have several applications in robotics, spanning context-adaptive, safe and social robots, wearable devices, regulatory processes and neurotechnology. AIF brings several things to the table in this setting. Perhaps the most important aspects are: (i) a commitment to an explicit, explainable and interpretable world model-in the form of a forward or generative model - that underwrites inference and learning, (ii) framing state estimation, control and planning as different aspects of the same inverse or inference problem, whose solution affords context sensitivity and robustness (iii) and, finally, supplying a tractable objective function that subsumes different kinds of (Bayes) optimality: namely, an expected free energy that subsumes Bayesian decision theory and Bayesian optimal design [2,32]. The latter brings with it a quintessentially belief-based specification of sentient behaviour that can be read as equipping robots with the right kind of curiosity. These foundational features of AIF are, we suppose, also found in human subjects, and therefore place AIF robots in a potentially more empathetic relationship to their human operators. It will be interesting to see whether-or how-these features are leveraged over the next few years.

In short, AIF is generally considered to endow robots and artificial agents with adaptive capabilities. While promising, the application is in its early days and much work remains to be undertaken in order to resolve practical challenges and fulfil the framework's potential. Current endeavours include scaling AIF to handle high dimensional state-spaces in a variety of applications $[10,12,13,59]$, effectively learning the generative model from data $[2,34]$, and show its practicality in the real world, beyond the lab boundaries. While significant engineering challenges remain, the state-of-the-art laboratory experiments show AIF's potential as a powerful method in robotics [14].

Author Contributions: Conceptualization, L.D.C., P.L., N.S., K.F. and S.K.; writing-original draft preparation, L.D.C., P.L. and S.K.; writing-review and editing, N.S. and K.F.; visualization, P.L. and N.S.; supervision, K.F. All authors have read and agreed to the published version of the manuscript.

Funding: L.D.C. is supported by the Fonds National de la Recherche, Luxembourg (Project code: 13568875). P.L. is supported by Spikeference project, Human Brain Project Specific Grant Agreement 3 (ID: 945539). N.S. is funded by the Medical Research Council (MR/S502522/1) and 2021-2022 Microsoft PhD Fellowship. K.F. is supported by funding for the Wellcome Centre for Human Neuroimaging (Ref: 205103/Z/16/Z) and a Canada-UK Artificial Intelligence Initiative (Ref: ES/T01279X/1). This publication is based on work partially supported by the EPSRC Centre for Doctoral Training in Mathematics of Random Systems: Analysis, Modelling and Simulation (EP/S023925/1).

Data Availability Statement: Not applicable.

Acknowledgments: The authors thank Jeroen Infographics for designing the Figure. The authors thank Areeb Mian and Sima Al-Asad for helpful input on a previous version of the manuscript.

Conflicts of Interest: The authors declare no conflict of interest.

\section{References}

1. Da Costa, L.; Parr, T.; Sajid, N.; Veselic, S.; Neacsu, V.; Friston, K. Active inference on discrete state-spaces: A synthesis. J. Math. Psychol. 2020, 99, 102447. [CrossRef] [PubMed]

2. Barp, A.; Da Costa, L.; França, G.; Friston, K.; Girolami, M.; Jordan, M.I.; Pavliotis, G.A. Geometric Methods for Sampling, Optimisation, Inference and Adaptive Agents. In Geometry and Statistics; Academic Press: Cambridge, MA, USA, 2022.

3. Friston, K.J.; Daunizeau, J.; Kilner, J.; Kiebel, S.J. Action and behavior: A free-energy formulation. Biol. Cybern. 2010, 102, 227-260. [CrossRef] [PubMed]

4. Buckley, C.L.; Kim, C.S.; McGregor, S.; Seth, A.K. The free energy principle for action and perception: A mathematical review. J. Math. Psychol. 2017, 81, 55-79. [CrossRef]

5. Friston, K. The free-energy principle: A unified brain theory? Nat. Rev. Neurosci. 2010, 11, 127-138. [CrossRef] [PubMed] 
6. Friston, K.; Kilner, J.; Harrison, L. A free energy principle for the brain. J. Physiol. 2006, 100, 70-87. [CrossRef] [PubMed]

7. Friston, K.; Da Costa, L.; Sajid, N.; Heins, C.; Ueltzhöffer, K.; Pavliotis, G.A.; Parr, T. The free energy principle made simpler but not too simple. arXiv 2022, arXiv:2201.06387.

8. Parr, T. The Computational Neurology of Active Vision. Ph.D. Thesis, University College London, London, UK, 2019.

9. Millidge, B. Deep active inference as variational policy gradients. J. Math. Psychol. 2020, 96, 102348. [CrossRef]

10. Fountas, Z.; Sajid, N.; Mediano, P.A.M.; Friston, K. Deep active inference agents using Monte-Carlo methods. arXiv 2020, arXiv:2006.04176.

11. Tschantz, A.; Millidge, B.; Seth, A.K.; Buckley, C.L. Reinforcement Learning through Active Inference. arXiv 2020, arXiv:2002.12636.

12. Sajid, N.; Tigas, P.; Zakharov, A.; Fountas, Z.; Friston, K. Exploration and preference satisfaction trade-off in reward-free learning. arXiv 2021, arXiv:2106.04316.

13. Mazzaglia, P.; Verbelen, T.; Dhoedt, B. Contrastive Active Inference. Available online: https://openreview.net/forum?id=5t5 FPwzE6mq (accessed on 18 February 2022).

14. Lanillos, P.; Meo, C.; Pezzato, C.; Meera, A.A.; Baioumy, M.; Ohata, W.; Tschantz, A.; Millidge, B.; Wisse, M.; Buckley, C.L.; et al. Active Inference in Robotics and Artificial Agents: Survey and Challenges. arXiv 2021, arXiv:2112.01871.

15. Blei, D.M.; Kucukelbir, A.; McAuliffe, J.D. Variational Inference: A Review for Statisticians. J. Am. Stat. Assoc. 2017, 112, 859-877. [CrossRef]

16. Meo, C.; Lanillos, P. Multimodal VAE Active Inference Controller. In Proceedings of the 2021 IEEE/RSJ International Conference on Intelligent Robots and Systems (IROS), Prague, Czech, 27 September-1 October 2021; pp. 2693-2699. [CrossRef]

17. Silver, D.; Huang, A.; Maddison, C.J.; Guez, A.; Sifre, L.; van den Driessche, G.; Schrittwieser, J.; Antonoglou, I.; Panneershelvam, V.; Lanctot, M.; et al. Mastering the game of Go with deep neural networks and tree search. Nature 2016, 529, 484-489. [CrossRef] [PubMed]

18. Vinyals, O.; Babuschkin, I.; Czarnecki, W.M.; Mathieu, M.; Dudzik, A.; Chung, J.; Choi, D.H.; Powell, R.; Ewalds, T.; Georgiev, P.; et al. Grandmaster level in StarCraft II using multi-agent reinforcement learning. Nature 2019, 575, 350-354. [CrossRef] [PubMed]

19. Van Der Himst, O.; Lanillos, P. Deep Active Inference for Partially Observable MDPs. In Active Inference, IWAI 2020, Communications in Computer and Information Science; Verbelen, T., Lanillos, P., Buckley, C.L., De Boom, C., Eds.; Springer: Cham, Switzerland, 2020; Volume 1326. [CrossRef]

20. Cullen, M.; Davey, B.; Friston, K.J.; Moran, R. Active Inference in OpenAI Gym: A Paradigm for Computational Investigations Into Psychiatric Illness. Biol. Psychiatry Cogn. Neurosci. Neuroimaging 2018, 3, 809-818. [CrossRef] [PubMed]

21. Sajid, N.; Ball, P.].; Parr, T.; Friston, K.J. Active Inference: Demystified and Compared. Neural Comput. 2021, 33, 674-712. [CrossRef]

22. Marković, D.; Stojić, H.; Schwöbel, S.; Kiebel, S.J. An empirical evaluation of active inference in multi-armed bandits. Neural Netw. 2021, 144, 229-246. [CrossRef]

23. Paul, A.; Sajid, N.; Gopalkrishnan, M.; Razi, A. Active Inference for Stochastic Control. arXiv 2021, arXiv:2108.12245.

24. Friston, K.; Da Costa, L.; Hafner, D.; Hesp, C.; Parr, T. Sophisticated Inference. Neural Comput. 2021, 33, 713-763. [CrossRef]

25. Smith, R.; Friston, K.J.; Whyte, C.J. A step-by-step tutorial on active inference and its application to empirical data. J. Math. Psychol. 2022, 107, 102632. [CrossRef]

26. Friston, K.; Trujillo-Barreto, N.; Daunizeau, J. DEM: A variational treatment of dynamic systems. NeuroImage 2008, 41, 849-885. [CrossRef] [PubMed]

27. Meera, A.A.; Wisse, M. Free Energy Principle Based State and Input Observer Design for Linear Systems with Colored Noise. In Proceedings of the 2020 American Control Conference (ACC), Denver, CO, USA, 1-3 July 2020; pp. 5052-5058. [CrossRef]

28. Baltieri, M.; Isomura, T. Kalman filters as the steady-state solution of gradient descent on variational free energy. arXiv 2021, arXiv:2111.10530.

29. da Costa, L.; Sajid, N.; Parr, T.; Friston, K.; Smith, R. The relationship between dynamic programming and active inference: The discrete, finite-horizon case. arXiv 2020, arXiv:2009.08111.

30. Imohiosen, A.; Watson, J.; Peters, J. Active Inference or Control as Inference? A Unifying View. arXiv 2020, arXiv:2010.00262.

31. Millidge, B.; Tschantz, A.; Seth, A.K.; Buckley, C.L. On the Relationship Between Active Inference and Control as Inference. In International Workshop on Active Inference; Springer: Cham, Switzerland, 2020; pp. 3-11.

32. Sajid, N.; da Costa, L.; Parr, T.; Friston, K. Active inference, Bayesian optimal design, and expected utility. arXiv 2021, arXiv:2110.04074.

33. Smith, R.; Schwartenbeck, P.; Parr, T.; Friston, K. An Active Inference Approach to Modeling Structure Learning: Concept Learning as an Example Case. Front. Comput. Neurosci. 2020, 14, 41. [CrossRef]

34. Çatal, O.; Wauthier, S.; De Boom, C.; Verbelen, T.; Dhoedt, B. Learning Generative State Space Models for Active Inference. Front. Comput. Neurosci. 2020, 14, 103. [CrossRef]

35. Friston, K.J.; Lin, M.; Frith, C.D.; Pezzulo, G.; Hobson, J.A.; Ondobaka, S. Active Inference, Curiosity and Insight. Neural Comput. 2017, 29, 2633-2683. [CrossRef]

36. Oliver, G.; Lanillos, P.; Cheng, G. An empirical study of active inference on a humanoid robot. IEEE Trans. Cogn. Dev. Syst. 2021, 4, 1-17. [CrossRef]

37. Meera, A.A.; Wisse, M. Dynamic Expectation Maximization Algorithm for Estimation of Linear Systems with Colored Noise. Entropy 2021, 23, 1306. [CrossRef] 
38. Baltieri, M.; Buckley, C.L. PID Control as a Process of Active Inference with Linear Generative Models. Entropy 2019, 21, 257. [CrossRef] [PubMed]

39. Lanillos, P.; Cheng, G. Adaptive Robot Body Learning and Estimation Through Predictive Coding. In Proceedings of the 2018 IEEE/RSJ International Conference on Intelligent Robots and Systems (IROS), Madrid, Spain, 1-5 October 2018; pp. 4083-4090. [CrossRef]

40. Pezzato, C.; Ferrari, R.M.G.; Corbato, C.H. A Novel Adaptive Controller for Robot Manipulators Based on Active Inference. IEEE Robot. Autom. Lett. 2020, 5, 2973-2980. [CrossRef]

41. Chame, H.F.; Tani, J. Cognitive and motor compliance in intentional human-robot interaction. In Proceedings of the 2020 IEEE International Conference on Robotics and Automation (ICRA), Paris, France, 31 May-31 August 2020; pp. 11291-11297. [CrossRef]

42. Friston, K.; Moran, R.J.; Nagai, Y.; Taniguchi, T.; Gomi, H.; Tenenbaum, J. World model learning and inference. Neural Netw. 2021, 144, 573-590. [CrossRef] [PubMed]

43. Taniguchi, T.; Piater, J.; Worgotter, F.; Ugur, E.; Hoffmann, M.; Jamone, L.; Nagai, T.; Rosman, B.; Matsuka, T.; Iwahashi, N.; et al Symbol Emergence in Cognitive Developmental Systems: A Survey. IEEE Trans. Cogn. Dev. Syst. 2018, 11, 494-516. [CrossRef]

44. Pearl, J. Graphical Models for Probabilistic and Causal Reasoning. In Quantified Representation of Uncertainty and Imprecision; Smets, P., Ed.; Springer: Dordrecht, The Netherlands, 1998; pp. 367-389. [CrossRef]

45. Friston, K.J.; Parr, T.; De Vries, B. The graphical brain: Belief propagation and active inference. Netw. Neurosci. 2017, 1, 381-414 [CrossRef]

46. Verbelen, T.; Lanillos, P.; Buckley, C.; Boom, C.D. (Eds.) Active Inference. In Proceedings of the First International Workshop, IWAI 2020, Co-Located with ECML/PKDD 2020, Ghent, Belgium, 14 September 2020; Springer: Berlin/Heidelberg, Germany, 2020. [CrossRef]

47. Wirkuttis, N.; Tani, J. Leading or Following? Dyadic Robot Imitative Interaction Using the Active Inference Framework. IEEE Robot. Autom. Lett. 2021, 6, 6024-6031. [CrossRef]

48. Horii, T.; Nagai, Y. Active Inference Through Energy Minimization in Multimodal Affective Human-Robot Interaction. Front. Robot. AI 2021, 8, 684401. [CrossRef]

49. Lanillos, P.; Pages, J.; Cheng, G. Robot Self/Other Distinction: Active Inference Meets Neural Networks Learning in a Mirror. In Proceedings of the ECAI 2020-24th European Conference on Artificial Intelligence, Compostela, Spain, 29 August-8 September 2020; IOS Press: Amsterdam, The Netherlands, 2020; pp. 2410-2416. [CrossRef]

50. Shin, J.Y.; Kim, C.; Hwang, H.J. Prior preference learning from experts: Designing a reward with active inference. Neurocomputing 2021, 12, 42. [CrossRef]

51. Friston, K. Complexity and Computation in the Brain: The Knowns and the Known Unknowns. Available online: https: / / direct.mit.edu/books/book/4588/chapter/204732/Complexity-and-Computation-in-the-Brain-The-Knowns (accessed on 18 February 2022).

52. Lanillos, P.; van Gerven, M. Neuroscience-inspired perception-action in robotics: Applying active inference for state estimation, control and self-perception. arXiv 2021, arXiv:2105.04261.

53. Ajoudani, A.; Zanchettin, A.M.; Ivaldi, S.; Albu-Schäffer, A.; Kosuge, K.; Khatib, O. Progress and prospects of the human-robot collaboration. Auton. Robot. 2018, 42, 957-975. [CrossRef]

54. Çatal, O.; Verbelen, T.; Van de Maele, T.; Dhoedt, B.; Safron, A. Robot navigation as hierarchical active inference. Neural Netw. 2021, 142, 192-204. [CrossRef]

55. Tschantz, A.; Barca, L.; Maisto, D.; Buckley, C.L.; Seth, A.K.; Pezzulo, G. Simulating homeostatic, allostatic and goal-directed forms of interoceptive control using active inference. Biol. Psychol. 2022, 169, 108266. [CrossRef]

56. Friston, K.J.; Fitzgerald, T.H.B.; Rigoli, F.; Schwartenbeck, P.; Pezzulo, G. Active Inference: A Process Theory. Neural Comput. 2017, 29, 1-49. [CrossRef]

57. Cheng, G.; Ehrlich, S.K.; Lebedev, M.; Nicolelis, M.A.L. Neuroengineering challenges of fusing robotics and neuroscience. Sci. Robot. 2020, 5, eabd1911. [CrossRef]

58. Sajid, N.; Parr, T.; Gajardo-Vidal, A.; Price, C.J.; Friston, K.J. Paradoxical lesions, plasticity and active inference. Brain Commun. 2020, 2, fcaa164. [CrossRef]

59. Tschantz, A.; Baltieri, M.; Seth, A.K.; Buckley, C.L. Scaling active inference. arXiv 2019, arXiv:1911.10601. 